\title{
Effects of estrogen deficiency on microstructural changes in rat alveolar bone proper and periodontal ligament
}

\author{
ZHONGSHUANG LIU ${ }^{1}$, LISHA LIU ${ }^{1}, \mathrm{CHEN} \mathrm{KANG}^{1}$, QI XIE ${ }^{2}, \mathrm{BIN} \mathrm{ZHANG}^{1,3}$ and YING $\mathrm{LI}^{1,3}$ \\ ${ }^{1}$ Sino-Russian Institute of Hard Tissue Development and Regeneration, \\ The Second Affiliated Hospital of Harbin Medical University; ${ }^{2}$ School of Stomatology, \\ Harbin Medical University; ${ }^{3}$ Heilongjiang Academy of Medical Sciences, Harbin, Heilongjiang 150001, P.R. China
}

Received July 10, 2014; Accepted March 16, 2015

DOI: $10.3892 / \mathrm{mmr} .2015 .3891$

\begin{abstract}
The present study aimed to analyze the effects of estrogen deficiency on buccal alveolar bone proper and the periodontal ligament in ovariectomized (OVX) rats, compared with rats that had been subjected to sham treatment. Morphological and histological changes in the periodontium were analyzed using micro-computed tomography and paraffin sectioning. Sections were stained using hematoxylin and eosin, and tartrate-resistant acid phosphatase. Expression of receptor activator of nuclear factor- $\kappa \mathrm{B}$ ligand (RANKL), dentin matrix protein $1 \mathrm{C}$-terminal (DMP1-C) and osteopontin (OPN) were analyzed using immunohistochemistry. Histomorphometric analysis of buccal alveolar bone proper samples revealed porotic changes and disorganized bone structure in OVX rats. Furthermore, bone mineral density and pore spacing were significantly lower in OVX rats compared with sham rats. Porosity was significantly higher in OVX rats compared with sham rats $(\mathrm{P}<0.01)$. A greater number of osteoclasts were observed along the margins of the buccal alveolar bone proper samples from OVX rats compared with those from the sham rats. Expression of OPN and RANKL was significantly higher, and that of DMP1-C was significantly lower, in OVX rats compared with sham rats. Ovariectomy-induced osteoporosis is capable of changing the structure of buccal alveolar bone proper and the periodontal ligament, which is likely to increase the risk of periodontal disease.
\end{abstract}

Correspondence to: Professor Bin Zhang or Dr Ying Li, Sino-Russian Institute of Hard Tissue Development and Regeneration, The Second Affiliated Hospital of Harbin Medical University, 246 Xue Fu Road, Harbin, Heilongjiang 150001, P.R. China

E-mail: zhangbhyd@126.com

E-mail: bonnieli1122@gmail.com

Key words: ovariectomy, alveolar bone proper, periodontal ligament, micro-computed tomography, receptor activator of nuclear factor- $\kappa \mathrm{B}$ ligand, osteopontin, dentin matrix protein 1

\section{Introduction}

Cortical porosity and tissue mineral density are associated with the mechanical properties of bone, and exert significant effects on cortical bone quality. The alveolar bone proper is a type of cortical bone, the condition of which is an important prognostic factor for patients with periodontal diseases. Periodontitis is a chronic destructive disease, which is characterized by inflammation of the supporting tissues of the teeth, resulting in periodontal tissue damage and alveolar bone loss $(1,2)$.

Osteoporosis is a growing public health problem, which is characterized by bone mass reduction and micro-architectural deterioration in the bone (3). Osteoporosis is primarily observed in post-menopausal women, in response to a reduction in estrogen levels in the blood, which leads to excessive bone remodeling (4). Studies have shown that estrogen deficiency leads to bone loss in the long bones and vertebrae. However, the effects of estrogen deficiency on human alveolar bone proper remain unclear $(5,6,7)$.

Since osteoporosis and periodontal diseases are types of bone resorptive diseases, it is hypothesized that osteoporosis is associated with periodontal disease progression (8-11). However, certain studies have failed to demonstrate an association between estrogen deficiency and periodontitis, a prominent cause of tooth loss, in elderly patients (12-14). Moriya et al (13) demonstrated that periodontal destruction may not be caused by osteoporosis alone. Furthermore, a study by Kribbs et al (14) suggested that osteoporosis does not cause a reduction in alveolar crest height. However, the results of their study demonstrated that there may be an association between osteoporosis and residual ridge density. By contrast, a number of studies have reported a reduction in alveolar ridge height in patients with reduced bone mineral density (BMD) $(15,16)$. Dvorak et al $(17)$ showed that cortical porosity in the alveolar bone region was greater in osteoporotic sheep compared with healthy sheep, and these differences were more pronounced at the endosteal surfaces of the mandibular cortex.

The clinical diagnosis of periodontal disease is typically confirmed using dental x-rays and panoramic pantomograms, which are used in order to observe the changes in periodontal ligament width and alveolar crest height in 2D images. 
However, a finding of similar alveolar ridge height and periodontal ligament width in patients with and without osteoporosis, does not exclude changes in cortical bone structure, as these imaging techniques may not detect subtle changes. An improved understanding of the associations between alveolar bone microstructure and estrogen deficiency may improve methods for periodontal tissue evaluation in patients with periodontal disease.

The aim of the present study was to analyze buccal alveolar bone proper and periodontal ligament changes in ovariectomized (OVX) rats and to clarify the associations between osteoporosis and periodontal disease. The current study may provide novel insights into the prevention and diagnosis of post-menopausal women with periodontal disease.

\section{Materials and methods}

Animal experiments. Female Wistar rats $(\mathrm{n}=20$; age $=8$ weeks; Cyagen Biosciences, Guangzhou, China) were used in the present study. All rats were maintained under the identical conditions and fed on standard Rodent diet with free access to food and water. Animals were divided randomly: ten were subjected to ovariectomy (OVX group) in which the ovaries were removed bilaterally. The remaining ten animals received a sham surgery (sham group) in which the ovaries were exposed and some of the fat tissues around the ovary were removed. Prior to the experiments, protocols were approved by the Ethics Committee of Harbin Medical University (Heilongjiang, China) and experiments were performed according to the University's Guidelines for Animal Experimentation.

Tissue preparation and histological analysis. All rats were sacrificed using chloral hydrate (Sigma-Aldrich, St. Louis, MO, USA) at 12 weeks after surgery. In order to conduct the histological analysis, the rats were decapitated, mandible dissected out and any adhering soft tissue was removed and then fixed in $4 \%$ paraformaldehyde, at $4^{\circ} \mathrm{C}$ overnight. Following a wash phase using phosphate-buffered saline, the specimens were decalcified in $10 \%$ ethylenediaminetetraacetic acid for 2-4 weeks, dehydrated and embedded in paraffin using standard histological procedures, according to the methods described in a previous study (18). Serial sections $(4-\mu \mathrm{m})$ were prepared. Sections were subjected to hematoxylin and eosin (H\&E) staining, tartrate-resistant acid phosphatase (TRAP) staining and immunohistochemical (IHC) analysis.

TRAP staining. In order to detect osteoclast activity, TRAP staining was performed, according to the methods described in a previous study $(19,20)$. Paraffin sections $(4-\mu \mathrm{m})$ were deparaffinized using xylene (Beijing Zhongshan Golden Bridge, Beijing, China) and then rehydrated. Following rehydration, samples were placed in $0.2 \mathrm{M}$ acetate buffer (Beijing Zhongshan Golden Bridge) for $20 \mathrm{~min}$, at room temperature. Subsequently, sections were incubated for $30-60 \mathrm{~min}$ at $37^{\circ} \mathrm{C}$ in a mixture containing $0.8 \%$ naphthol AS-BI phosphate (Sigma-Aldrich, St Louis, MO, USA), 0.7\% fast red violet salt (Sigma-Aldrich) and $50 \mathrm{mM}$ sodium tartate, diluted

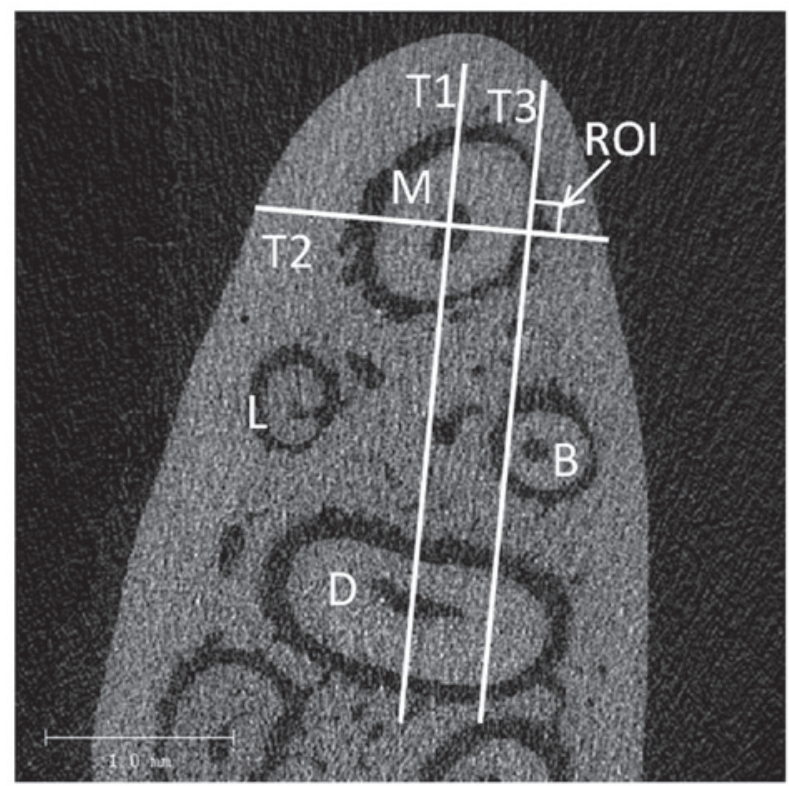

Figure 1. Micro-computed tomography images of a rat mandibular alveolar bone, indicating the ROI. Distance between T1 and T3=500 $\mu \mathrm{m}$. A $200 \times 200 \times 200-\mu \mathrm{m}$ cube was prepared for analyses with the T2 and T3 surfaces at the base. T1, sagittal plane passing through the centers of M1 mesial and distal roots; T2, coronal plane that penetrates the center of the M1 medial root; T3, sagittal surface parallel to T1 and perpendicular to T2; $\mathrm{M}$, mesial root; $\mathrm{B}$, buccal root; $\mathrm{D}$, distal root; $\mathrm{L}$, lingual root; ROI, region of interest.

in 0.2 $\mathrm{M}$ acetate buffer (pH 5.4), until osteoclasts appeared red. Samples were then counter-stained using hematoxylin, air dried and examined using a light microscope (Nikon 80i; Nikon Corporation, Tokyo, Japan). Osteoclasts were counted along the margins of the buccal alveolar bone proper in TRAP-stained sections.

IHC. IHC was performed using Diaminobenzidine Detection Kit (cat. no. PV-6000-D; Beijing Zhongshan Golden Bridge), according to the manufacturer's instructions (21-23). Tissue slices were dewaxed in xylene and rehydrated. Endogenous peroxidases were inhibited by incubation in $0.3 \%$ peroxide for $30 \mathrm{~min}$ at room temperature, followed by repeated washing in PBS. All sections were treated with 5\% normal goat serum (cat. no. ZLI-9022; Beijing Zhongshan Golden Bridge) for $30 \mathrm{~min}$ at room temperature. The sections were subsequently incubated overnight at $4{ }^{\circ} \mathrm{C}$ with one of the following primary antibodies: Rabbit anti-OPN polyclonal antibody (1:200; cat. no. ab8448; Abcam, Cambridge, MA, USA), rabbit anti-DMP1-C polyclonal antibody (1:500; cat. no. M176; Takara Bio, Inc., Otsu, Japan) and mouse anti-RANKL monoclonal antibody (1:800; cat. no. ALX-804-244-C100, Alexis Biochemicals, San Diego, CA, USA), followed by washing with PBS. According to the PV-6000-D direction, the sections were incubated with the secondary antibodies (goat anti-rabbit/mouse immunoglobulin G). Antibody complexes were visualized by the addition of a buffered diaminobenzidine substrate for $3 \mathrm{~min}$ and the reaction was inhibited by rinsing the sections in PBS. The intensity of immunostaining was assessed, according to the previously described method (23). 

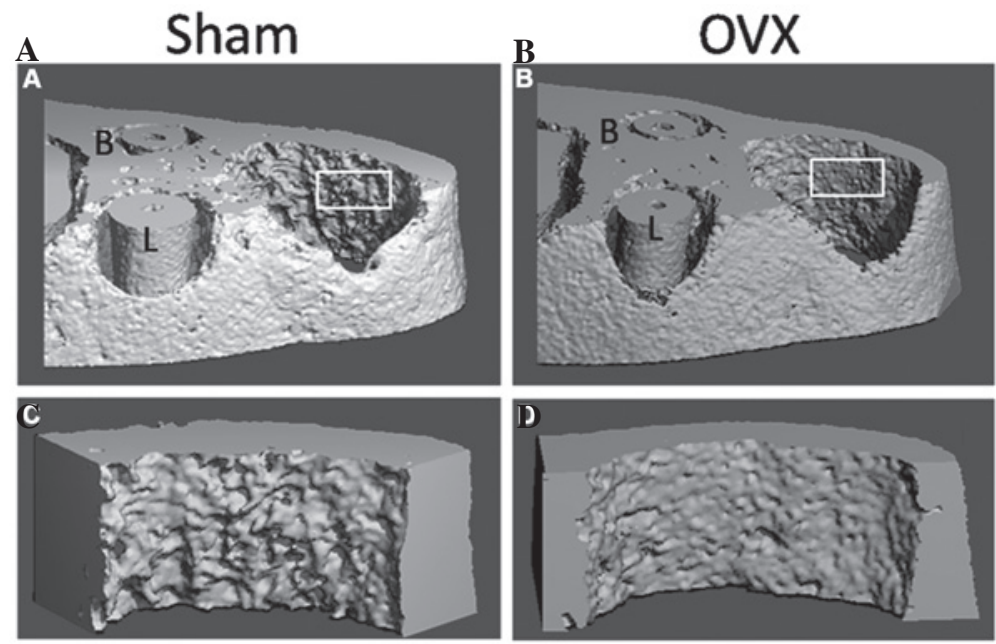

Figure 2. Representative images illustrating micro-computed tomography image analysis in buccal alveolar bone proper of the M1 mesial root. (A) and (C) represent the sham rats, and (B) and (D) represent OVX rats. (C) and (D) are of higher magnification, compared with those of (A) and (B). In OVX rats, a higher degree of bone remodeling was observed on the surface of buccal alveolar bone proper compared with that of sham rats. B, buccal root; L, lingual root; OVX, ovariectomized.
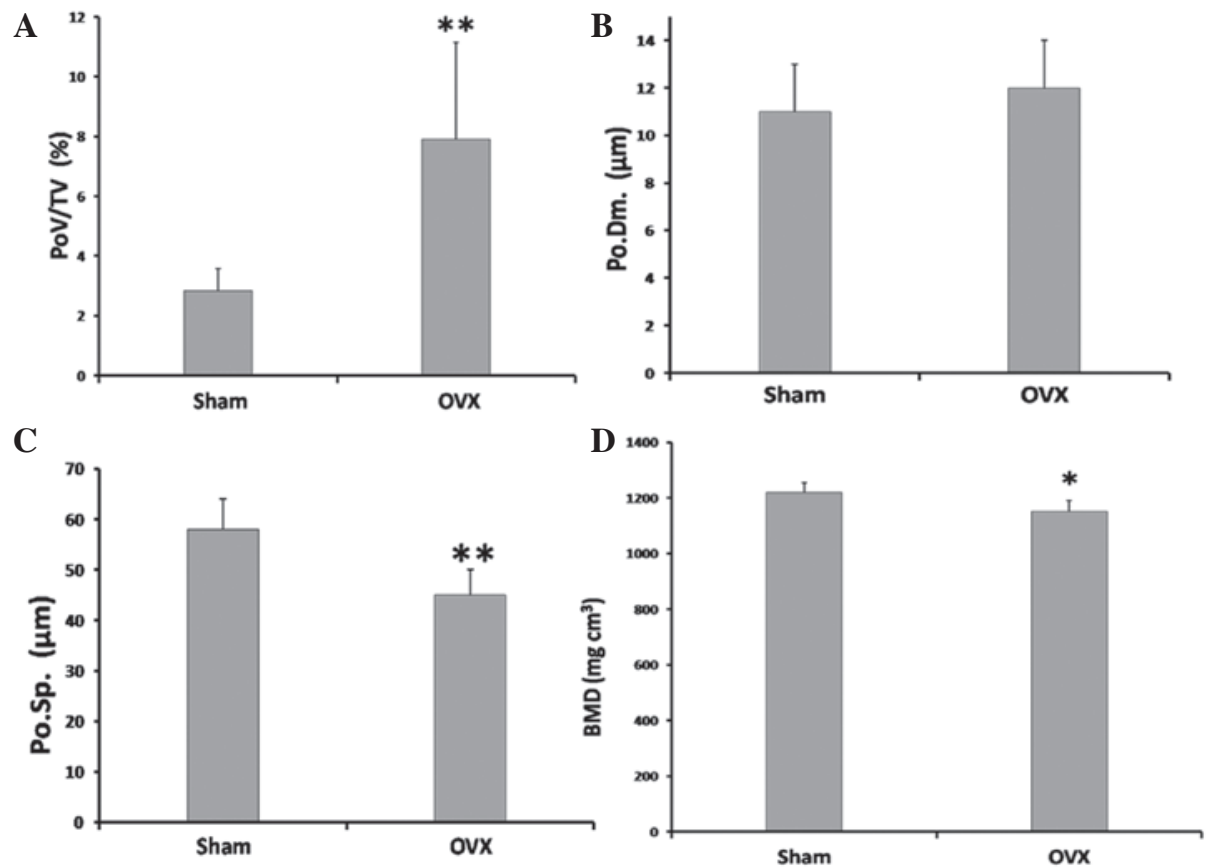

Figure 3. Micro-computed tomography histomorphometric comparisons between sham and OVX rats. (A) PoV/TV, (B) Po.Dm., (C) Po.Sp., and (D) BMD. Values represent the mean \pm standard deviation. ${ }^{*} \mathrm{P}<0.05$ and ${ }^{* *} \mathrm{P}<0.01$, compared with sham rats. PoV/TV, porosity (pore volume/total volume); Po.Dm., pore diameter; Po.Sp., pore spacing; BMD, bone mineral density; OVX, ovariectomized rats.

Micro-computed tomography (micro-CT) scanning and alveolar bone analysis. Samples were analyzed using a Scanco Medical micro-CT 35 (Scanco Medical AG, Bassersdorf, Switzerland) with the following parameters: $6-\mu \mathrm{m}$ voxels, 114 milliamperes, 70 peak kilovoltage and an exposure time of 300 milliseconds. 3D reconstructions were performed in order to assess the morphological characteristics of the rat mandibles. Alveolar bone histomorphometrics were measured directly from the region of interest (ROI).

The ROI was manually established in the buccal alveolar bone proper of the mandibular first molar (M1). The sampling regions of the buccal alveolar bone proper from the mesial roots of the M1 were standardized using the following characteristics: A sagittal plane (T1), which passes the center of the M1 mesial and distal roots; a coronal plane (T2), which penetrates the center of M1 medial root; and a sagittal surface (T3), which represents the observation surface and is parallel to $\mathrm{T} 1$ and perpendicular to $\mathrm{T} 2$. The distance between $\mathrm{T} 1$ and T3 was $500 \mu \mathrm{m}$. Cubes of 200x200x200 $\mu \mathrm{m}$, with T2 and T3 surfaces as their bases, were then selected for analysis (Fig. 1). Cube size used in the present study was selected according to the methods described in a previous study (24). The following 


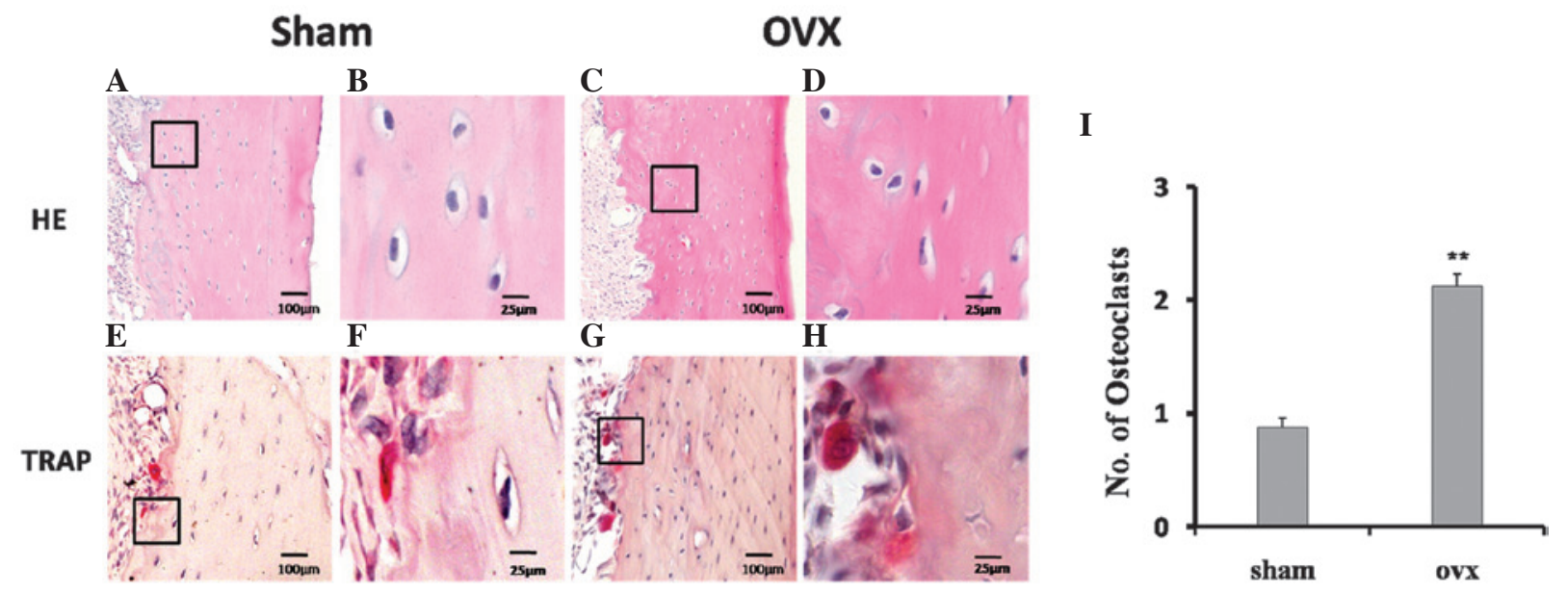

Figure 4. H\&E staining and TRAP staining of alveolar bones from sham and OVX rats. (A)-(D) were subjected to H\&E staining, and (E)-(H) were subjected to TRAP staining. (A), (B), (E) and (F) were obtained from sham rats, while (C), (D), (G) and (H) were obtained from OVX rats. (B), (D), (F) and (H) represent higher magnification images of the black squares shown in (A), (C), (E) and (G). (B) A healthy lamellar bone structure was observed in the buccal alveolar bone of sham rats. (D) Compared with sham rats, the buccal alveolar bone from OVX rats exhibited larger osteocyte lacunae and smaller lamellar bone structure. (E) and (G) Osteoclasts are multinuclear cells that appear red following TRAP staining and form a line along the margin of the buccal alveolar bone proper. (magnification of A, C, E and G, x100; magnification of B, D, F and H, x400). (I) A significant increase in TRAP-positive cells was observed along the margin of the buccal alveolar bone proper when compared with the controls. TRAP, tartrate-resistant acid phosphatase; OVX, ovariectomy group; H\&E, hematoxylin and eosin.

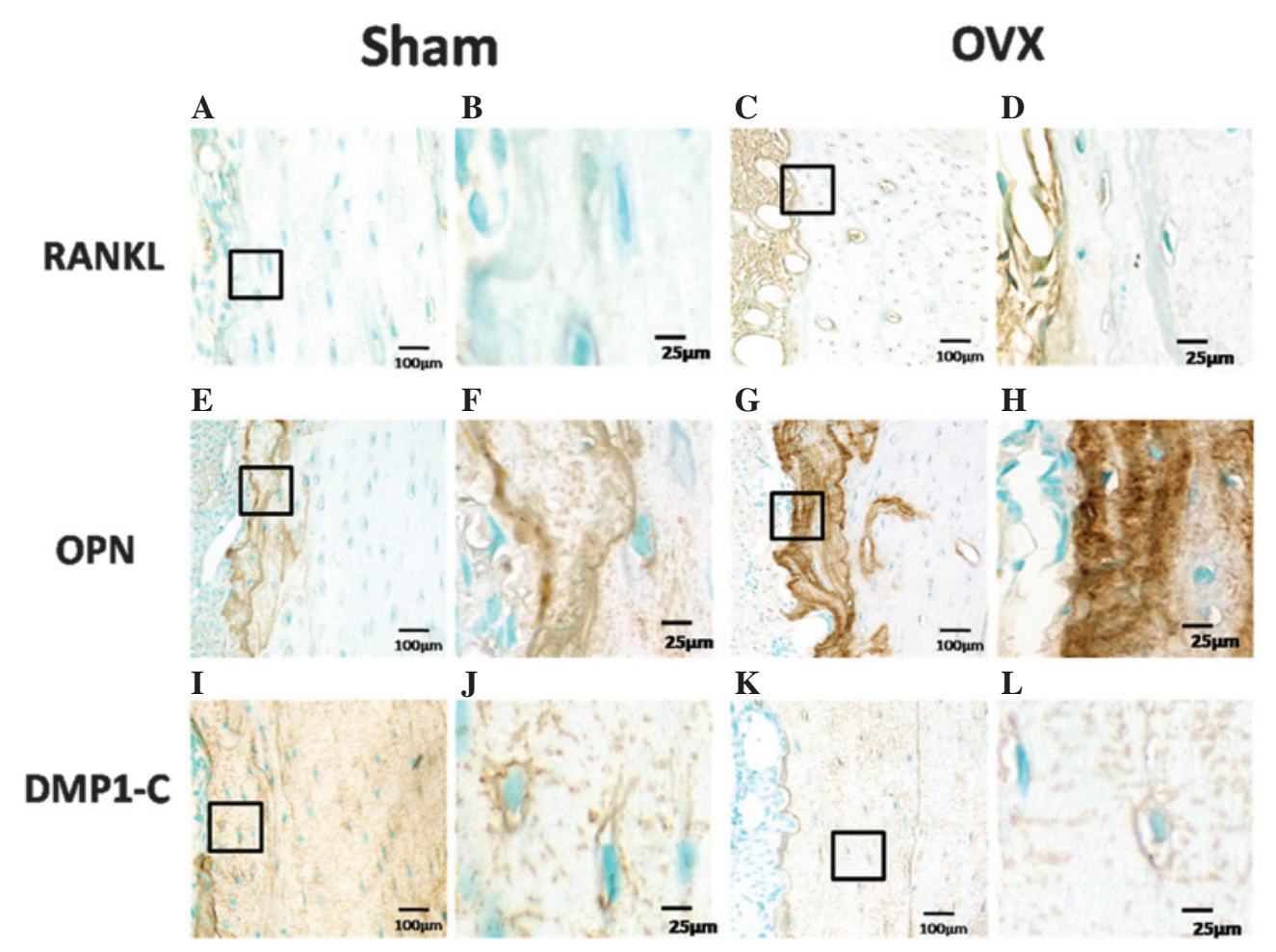

Figure 5. Representative immunohistochemical images of alveolar bone in samples from the mandibular first molar. (A), (B), (E), (F), (I) and (J) were obtained from sham rats and (C), (D), (G), (H), (K) and (L) from OVX rats. (B), (D), (F), (H), (J) and (L) represent higher magnification images of the black squares shown in (A), (C), (E), (G), (I) and (K). (A)-(D) IHC for RANKL; (E)-(H) IHC for OPN; (I)-(L) IHC for DMP1-C. RANKL was predominantly expressed in the cytoplasm of osteoblast and periodontal ligament cells in sham and OVX rats. (D) OVX rats exhibited high levels of RANKL expression, in particular in the periodontal ligament, adjacent to alveolar bone proper, compared with sham rats. The distribution of OPN expression was similar in sham and OVX rats. (E) and (G) OPN was predominantly observed in areas containing newly formed bone from sham and OVX rats. (F) and (H) A relatively strong signal for OPN was observed in the alveolar bone proper in OVX rats, while the signal for OPN was generally weak in rats in the sham group, compared with the OVX rats. DMP1-C expression was observed in the extracellular matrix surrounding osteocyte lacunae of newly formed bone samples. In sham rats, DMP1-C expression was higher compared with that in the OVX rats. (magnification of A, C, E, G, I and K, x100; magnification of B, D, F, H, J and L, x400). OVX, ovariectomy group; IHC, immunohistochemistry; RANKL, receptor activator of nuclear factor $\mathrm{kB}$ ligand; OPN, osteopontin; DMP1-C; dentin matrix protein $1 \mathrm{C}$-terminal.

3D micro-architecture parameters of the ROI were evaluated: BMD; porosity (pore volume/total volume; \%); pore diameter $(\mathrm{mm})$; and pore spacing $(\mathrm{mm})$.
Statistical analysis. Data are presented as the mean \pm standard deviation. Statistical analyses were conducted using SPSS version 13 (SPSS Inc., Chicago, IL, USA). Sham and OVX 
Table I. Micro-architectural measurements in the buccal alveolar bone proper of the mesial root of the mandibular first molar.

\begin{tabular}{lccc}
\hline Measurement & Sham & OVX & P-value \\
\hline PoV/TV $(\%)$ & $2.84 \pm 0.73$ & $7.90 \pm 3.25$ & $0.006^{\mathrm{b}}$ \\
Po.Dm. $(\mu \mathrm{m})$ & $11.00 \pm 2.00$ & $12.00 \pm 2.00$ & 0.112 \\
Po.Sp. $(\mu \mathrm{m})$ & $58.00 \pm 6.00$ & $45.00 \pm 5.00$ & $0.001^{\mathrm{b}}$ \\
BMD $\left.(\mathrm{mg} \mathrm{cm})^{3}\right)$ & $1219.05 \pm 36.12$ & $1150.49 \pm 40.28$ & $0.014^{\mathrm{a}}$ \\
\hline
\end{tabular}

${ }^{\mathrm{a}} \mathrm{P}<0.05$ and ${ }^{\mathrm{b}} \mathrm{P}<0.01$. PoV/TV, porosity (pore volume/total volume); Po.Dm., pore diameter; Po.Sp., pore spacing; BMD, bone mineral density. OVX, ovariectomized rats.

groups were compared using t-tests and $\mathrm{P}<0.05$ was considered to indicate a statistically significant difference.

\section{Results}

Micro-CT histomorphometry analysis of alveolar bone structure. 3D images obtained using micro-CT scanning were used to visualize the ROI in buccal alveolar bone proper samples from OXV and sham rats (Fig. 1). A greater degree of bone remodeling was observed on the surface of buccal alveolar bone proper samples from OVX rats compared with those of the sham rats (Figs. 2 and 3). Table I summarizes the morphological values measured using micro-CT. Porosity was significantly higher in OVX rats compared with sham rats $(\mathrm{P}=0.006)$. A higher density of pores was observed on the surfaces of buccal alveolar bone proper in OVX rats compared with those of the sham rats (Fig. 3A). No significant difference in pore diameter was observed between OVX and sham rats (Fig. 3B). BMD and pore spacing were significantly lower in OVX rats compared with the sham rats $(\mathrm{P}=0.014$ and 0.001 , respectively; Fig. 3C and D).

H\&E staining and TRAP staining in alveolar bone samples from $O V X$ rats. A reduction of lamellar bone structure in buccal alveolar bone proper was observed in OVX rats. A greater number of osteocytes was observed in alveolar bone samples from OVX rats compared with that in sham rats (Fig. 4A,B). Furthermore, the lacunae of osteocytes were larger in OVX rats compared with those in sham rats (Fig. 4A-D). Moreover, the periodontal ligament structure became discontinuous and a greater number of pores were observed along the buccal alveolar bone proper in OVX rats compared with that of sham rats.

TRAP staining was performed in order to evaluate periodontal tissue remodeling in the buccal alveolar bone in OVX and sham rats. Osteoclasts were recognized as multinuclear cells, which appeared dark red following TRAP staining (Fig. 4E-H). The number of TRAP-positive cells observed along the margin of the buccal alveolar bone proper was significantly lower in OVX rats compared with the sham rats (Fig. 4I).

IHC analyses of RANKL, OPN and DMPI-C in the buccal alveolar bone and periodontal ligament in OVX rats. In order to confirm the involvement of the osteoclastogenic factor, RANKL, in the remodeling of periodontal tissues in OVX rats, an IHC assay was conducted. RANKL staining in periodontal tissues from sham rats was weak, compared with that in OVX rats (Fig. 5A-D). In particular, RANKL expression was high in the periodontal ligament, adjacent to alveolar bone proper in OVX rats (Fig. 5C and D).

Estrogen deficiency led to significantly higher OPN expression levels in the periodontal ligament and adjacent alveolar bone in OVX rats compared with sham rats. OPN expression was observed in regions of new bone formation in the sham rats (Fig. 5E and F). By contrast, the entire region of the alveolar bone exhibited high OPN expression in OVX rats, in particular in the un-mineralized regions surrounding the osteocytesin (Fig. 5G and H).

DMP1-C expression was localized to the mineralized bone areas. DMP1-C expression was markedly higher in the alveolar bone of the sham rats than that of the OVX rats (Fig. 5I-L). In addition, lower levels of DMP1-C expression were observed in the osteoid and hypomineralized region of the alveolar bone in OVX rats compared with that in sham rats.

\section{Discussion}

The purpose of the present study was to evaluate the influence of estrogen deficiency on the buccal alveolar bone proper and periodontal ligament in rats. The current study aimed to investigate the possible association between osteoporosis and periodontal disease, in order to develop effective techniques for the prevention and diagnosis of periodontal disease in postmenopausal women.

In the present study, the effects of estrogen deficiency on the alveolar bone proper and periodontal ligament in OVX rats were investigated. A number of microstructural differences were observed in the buccal alveolar bone proper between OVX and sham rats. Porosity levels were markedly higher in OVX rats compared with those of the sham rats. Pore spacing was significantly lower in OVX rats compared with sham rats. Bone formation and resorption levels were significantly higher in OVX rats compared with those in sham rats. Following periodontal bacterial infection, changes in the alveolar bone microstructure, are likely to lead to a higher level of jawbone resorption (11,25-27). Overall, the results of the present study demonstrate that estrogen deficiency may exert effects on alveolar bone metabolism and lead to an increased risk of periodontal disease.

Buccal alveolar bone proper mass was significantly lower in the OVX rats compared with that in the sham rats. Therefore, 
estrogen deficiency may affect the process of alveolar bone remodeling in OVX rat mandibles. Payne et al (28) indicated that estrogen deficiency in postmenopausal women is associated with a loss of alveolar bone density. A number of studies have demonstrated a positive correlation between levels of skeletal BMD expression and periodontal disease $(15,16,25)$. Wactawski-Wende et al (29) demonstrated an association between alveolar bone loss and periodontal disease in post-menopausal women. Therefore, estrogen deficiency may lead to periodontal disease in post-menopausal women. Low bone density levels and porotic changes were observed in alveolar bone samples from OVX rats compared with sham rats. Therefore, estrogen deficiency in alveolar bone proper may lead to osteoblast inhibition and osteoclast induction.

Estrogen deficiency may exert complex effects in bone samples. Estrogen levels have been reported to exert direct anabolic effects on bone cells (30-33). Osteoblasts differentiate into osteocytes, which promote bone formation. By contrast, multinucleated osteoclasts promote bone resorption (23). In the present study, expression levels of extracellular matrix proteins, including OPN and DMP1-C, were used in order to indicate osteoblast and osteoclast differentiation. RANK is a key signaling pathway involved in osteoclast differentiation. RANK receptors are expressed on the surface of osteoclast precursors. Osteoblasts produce RANKL, which coordinates bone remodelling via the stimulation of nearby osteoclasts, which subsequently stimulates adjacent osteoblasts (34).

In the present study, RANKL expression was higher in OVX rats compared with sham rats and, therefore, osteoclast activity was greater, which led to higher levels of alveolar bone loss and changes in alveolar bone structure. Furthermore, osteoclastogenesis has been shown to be induced in periodontium samples from OVX rats, suggesting that alveolar bone resorption may be associated with the increased osteoclast activity (35).

The periodontal ligament is located between the hard tissue, cementum and the alveolar bone, which are associated with periodontal development and regeneration. The results of the present study demonstrated that the periodontal ligament structure became discontinuous, and porotic changes were observed along the buccal alveolar bone proper in OVX rats compared with sham rats. Therefore, estrogen deficiency may lead to osteoclastogenesis induction via the upregulation of RANKL expression in periodontal ligament cells. Furthermore, estrogen-deficient periodontal ligament cells may be involved, not only in the maintenance and remodeling of the ligament, but also in the metabolism of adjacent alveolar bone tissue.

OPN is a multifunctional protein involved in bone turnover, osteoclastogenesis and osteoblast function. It is predominantly observed in the cement line and resorption sites (21). OPN activation in osteoblasts may accelerate the adhesion of osteoclasts and cause osteoclast activation, thereby increasing alveolar bone resorption. Furthermore, OVX-induced bone resorption using OPN-knockout in mice demonstrated that OPN is involved in bone remodeling (36-38). In the present study, OPN was expressed in the periodontal ligament and adjacent alveolar bone in OVX rats. This observation suggests that estrogen deficiency may led to an enhanced risk of periodontal destruction in OVX rats.
DMP1 is an acidic non-collagenous protein that is associated with osteogenesis and dentinogenesis. DMP1 predominantly occurs as proteolytically produced $37 \mathrm{kDa}$ (DMP1-N) and $57 \mathrm{kDa}$ (DMP1-C) fragments in the bone and dentine extracellular matrices (23). DMP1-N is expressed in un-mineralized predentin, while DMP1-C is predominantly expressed in mineralized dentine and bone tissue (39). Thus, DMP1-C is a marker for the mineralization of osteoid and immature bone formation. In the present study, the signal for DMP1-C in alveolar bone samples from OVX rats was markedly lower compared with samples from the sham rats. This suggested that the low expression of the DMP1-C in OVX rats may lead to low levels of osteogenesis in the alveolar bone. Therefore, bone remodeling in periodontal tissues is enhanced in estrogen-deficient rats.

In conclusion, estrogen deficiency may lead to an increase in RANKL and OPN expression levels, which may contribute to enhanced levels of osteoclast activation and migration. In addition, the results of the present study suggest that estrogen deficiency may lead to alveolar bone damage. Therefore, further research into the influence of estrogen deficiency on alveolar bones of post-menopausal women is required. In order to elucidate the effects of estrogen deficiency on periodontal disease, it is necessary to conduct further in vivo experiments using OVX animals.

\section{Acknowledgements}

This study was supported by grants from the Heilongjiang Postgraduate Innovation Science Foundation (grant no. YJSCX2012-240HLJ), the National Natural Science Foundation of China (grant no. 81170960), the Foundation of Department of Science and Technology of Heilongjiang Province (grant no. GC12C303-2) and the National Key Science Instrument and Equipment Development Project (grant no. 2011YQ04008708).

\section{References}

1. Irfan UM, Dawson DV and Bissada NF: Epidemiology of periodontal disease: A review and clinical perspectives. J Int Acad Periodontol 3: 14-21, 2001.

2. Michaud DS, Joshipura K, Giovannucci E and Fuchs CS: A prospective study of periodontal disease and pancreatic cancer in US male health professionals. J Natl Cancer Inst 99: 171-175, 2007.

3. Kanis JA, Melton LJ III, Christiansen C, Johnston CC and Khaltaev N: The diagnosis of osteoporosis. J Bone Miner Res 9: 1137-1141, 1994.

4. Genant HK, Cooper C, Poor G, Reid I, Ehrlich G, Kanis J, Nordin BE, Barrett-Connor E, Black D, Bonjour JP, et al: Interim report and recommendations of the World Health Organization task-force for osteoporosis. Osteoporos Int 10: 259-264, 1999.

5. Binte Anwar R, Tanaka M, Kohno S, Ikegame M, Watanabe N, Nowazesh Ali M and Ejiri S: Relationship between porotic changes in alveolar bone and spinal osteoporosis. J Dent Res 86: 52-57, 2007.

6. Bollen AM, Taguchi A, Hujoel PP and Hollender LG: Case-control study on self-reported osteoporotic fractures and mandibular cortical bone. Oral Surg Oral Med Oral Pathol Oral Radiol Endod 90: 518-524, 2000.

7. Kalu DN: The ovariectomized rat model of postmenopausal bone loss. Bone Miner 15: 175-191, 1991.

8. Wronski TJ, Dann LM, Qi H and Yen CF: Skeletal effects of withdrawal of estrogen and diphosphonate treatment in ovariectomized rats. Calcif Tissue Int 53: 210-216, 1993. 
9. Wronski TJ, Yen CF, Burton KW, Mehta RC, Newman PS, Soltis EE and DeLuca PP: Skeletal effects of calcitonin in ovariectomized rats. Endocrinology 129: 2246-2250, 1991.

10. Kribbs PJ: Comparison of mandibular bone in normal and osteoporotic women. J Prosthet Dent 63: 218-222, 1990.

11. Groen JJ, Menczel J and Shapiro S: Chronic destructive periodontal disease in patients with presenile osteoporosis. J Periodontol 39: 19-23, 1968.

12. Jagelaviciene E and Kubilius R: The relationship between general osteoporosis of the organism and periodontal diseases. Medicina (Kaunas) 42: 613-618, 2006.

13. Moriya Y, Ito K and Murai S: Effects of experimental osteoporosis on alveolar bone loss in rats. J Oral Sci 40: 171-175, 1998

14. Kribbs PJ, Smith DE and Chesnut CH III: Oral findings in osteoporosis. Part II: Relationship between residual ridge and alveolar bone resorption and generalized skeletal osteopenia. J Prosthet Dent 50: 719-724, 1983.

15. Klemetti E and Vainio P: Effect of bone mineral density in skeleton and mandible on extraction of teeth and clinical alveolar height. J Prosthet Dent 70: 21-25, 1993.

16. Bays RA and Weinstein RS: Systemic bone disease in patients with mandibular atrophy. J Oral Maxillofac Surg 40: 270-272, 1982.

17. Dvorak G, Reich KM, Tangl S, Goldhahn J, Haas R and Gruber R: Cortical porosity of the mandible in an osteoporotic sheep model. Clin Oral Implants Res 22: 500-505, 2011.

18. Fen JQ, Zhang J, Dallas SL, Lu Y, Chen S, Tan X, Owen M, Harris SE and MacDougall M: Dentin matrix protein 1, a target molecule for Cbfal in bone, is a unique bone marker gene. J Bone Miner Res 17: 1822-1831, 2002.

19. Ejiri S, Toyooka E, Tanaka M, Anwar RB and Kohno S: Histological and histomorphometrical changes in rat alveolar bone following antagonistic tooth extraction and/or ovariectomy. Arch Oral Biol 51: 941-950, 2006.

20. Kollet O, Dar A, Shivtiel S, Kalinkovich A, Lapid K, Sztainberg Y, Tesio M, Samstein RM, Goichberg P, Spiegel A, et al: Osteoclasts degrade endosteal components and promote mobilization of hematopoietic progenitor cells. Nat Med 12: 657-664, 2006.

21. Sodek J, Ganss B and McKee MD: Osteopontin. Crit Rev Oral Biol Med 11: 279-303, 2000.

22. Jaiprakash A, Prasadam I, Feng JQ, Liu Y, Crawford R and Xiao Y: Phenotypic characterization of osteoarthritic osteocytes from the sclerotic zones: A possible pathological role in subchondral bone sclerosis. Int J Biol Sci 8: 406-417, 2012.

23. Zhang B, Sun Y, Chen L, Guan C, Guo L and Qin C: Expression and distribution of SIBLING proteins in the predentin/dentin and mandible of hyp mice. Oral Dis 16: 453-464, 2010.

24. Tanaka M, Ejiri S, Toyooka E, Kohno S and Ozawa H: Effects of ovariectomy on trabecular structures of rat alveolar bone. J Periodontal Res 37: 161-165, 2002.

25. Bando K, Nitta H, Matsubara M and Ishikawa I: Bone mineral density in periodontally healthy and edentulous postmenopausal women. Ann Periodontol 3: 322-326, 1998.
26. Weyant RJ, Pearlstein ME, Churak AP, Forrest K, Famili P and Cauley JA: The association between osteopenia and periodontal attachment loss in older women. J Periodontol 70: 982-991, 1999.

27. Kribbs PJ, Chesnut CH III, Ott SM and Kilcoyne RF: Relationships between mandibular and skeletal bone in an osteoporotic population. J Prosthet Dent 62: 703-707, 1989.

28. Payne JB, Zachs NR, Reinhardt RA, Nummikoski PV and Patil K: The association between estrogen status and alveolar bone density changes in postmenopausal women with a history of periodontitis. J Periodontol 68: 24-31, 1997.

29. Wactawski-Wende J, Grossi SG, Trevisan M, Genco RJ, Tezal M, Dunford RG, Ho AW, Hausmann E and Hreshchyshyn MM: The role of osteopenia in oral bone loss and periodontal disease. J Periodontol 67 (Suppl 10): 1076-1084, 1996.

30. Majeska RJ, Ryaby JT and Einhorn TA: Direct modulation of osteoblastic activity with estrogen. J Bone Joint Surg Am 76: 713-721, 1994

31. Manolagas SC and Jilka RL: Bone marrow, cytokines, and bone remodeling. Emerging insights into the pathophysiology of osteoporosis. N Engl J Med 332: 305-311, 1995.

32. Pacifici R: Estrogen, cytokines, and pathogenesis of postmenopausal osteoporosis. J Bone Miner Res 11: 1043-1051, 1996.

33. Hughes DE, Dai A, Tiffee JC, Li HH, Mundy GR and Boyce BF: Estrogen promotes apoptosis of murine osteoclasts mediated by TGF-beta. Nat Med 2: 1132-1136, 1996.

34. Udagawa N, Takahashi N, Yasuda H, Mizuno A, Itoh K, Ueno Y, Shinki T, Gillespie MT, Martin TJ, Higashio K and Suda T: Osteoprotegerin produced by osteoblasts is an important regulator in osteoclast development and function. Endocrinology 141: 3478-3484, 2000.

35. Kawamoto S, Ejiri S, Nagaoka E and Ozawa H: Effects of oestrogen deficiency on osteoclastogenesis in the rat periodontium. Arch Oral Biol 47: 67-73, 2002.

36. Rajachar RM, Truong AQ and Giachelli CM: The influence of surface mineral and osteopontin on the formation and function of murine bone marrow-derived osteoclasts. J Mater Sci Mater Med 19: 3279-3285, 2008

37. Ono N, Nakashima K, Rittling SR, Schipani E, Hayata T, Soma K, Denhardt DT, Kronenberg HM, Ezura Y and Noda M: Osteopontin negatively regulates parathyroid hormone receptor signaling in osteoblasts. J Biol Chem 283: 19400-19409, 2008

38. Namkung-Matthai H, Appleyard R, Jansen J, Hao Lin J, Maastricht S, Swain M, Mason RS, Murrell GA, Diwan AD and Diamond T: Osteoporosis influences the early period of fracture healing in a rat osteoporotic model. Bone 28: 80-86, 2001.

39. Maciejewska I, Qin D, Huang B, Sun Y, Mues G, Svoboda K, Bonewald L, Butler WT, Feng JQ and Qin C: Distinct compartmentalization of dentin matrix protein 1 fragments in mineralized tissues and cells. Cells Tissues Organs 189: 186-191, 2009. 